\title{
Turbulent diffusion and galactic magnetism
}

\author{
Axel Brandenburg \& Fabio Del Sordo \\ NORDITA, Roslagstullsbacken 23, SE-10691 Stockholm, Sweden; and \\ Department of Astronomy, Stockholm University, SE-10691 Stockholm, Sweden
}

\begin{abstract}
Using the test-field method for nearly irrotational turbulence driven by spherical expansion waves it is shown that the turbulent magnetic diffusivity increases with magnetic Reynolds numbers. Its value levels off at several times the rms velocity of the turbulence multiplied by the typical radius of the expansion waves. This result is discussed in the context of the galactic mean-field dynamo.
\end{abstract}

Keywords. turbulence, (magnetohydrodynamics:) MHD, galaxies: magnetic fields

The galactic dynamo is believed to be powered by supernova-driven turbulence. This type of forcing does not directly produce vorticity; it can only be produced indirectly through oblique shocks, i.e. through the baroclinic term. The aim of this work is to assess whether vorticity is actually important for the dynamo.

The galactic magnetic field has a strong large-scale component which is generally believed to be due to a mean-field dynamo of $\alpha \Omega$ type that is governed by the equation

$$
\frac{\partial \overline{\boldsymbol{B}}}{\partial t}=\nabla \times\left(\overline{\boldsymbol{U}} \times \overline{\boldsymbol{B}}+\overline{E M F}-\eta \mu_{0} \overline{\boldsymbol{J}}\right), \quad \text { where } \quad \overline{e m f}_{i}=\alpha_{i j} \bar{B}_{j}-\eta_{i j} \mu_{0} \bar{J}_{j}
$$

is the mean electromotive force, $\overline{\boldsymbol{J}}=\boldsymbol{\nabla} \times \overline{\boldsymbol{B}} / \mu_{0}$ is the mean current density, $\overline{\boldsymbol{U}}$ is the mean flow, and $\mu_{0}$ is the vacuum permeability. In order to assess its efficiency, one needs to determine the tensors $\alpha_{i j}$ (the " $\alpha$ effect") and $\eta_{i j}$ (turbulent magnetic diffusivity). Note that $\alpha_{i j}$ is a pseudo tensor and non-vanishing diagonal components can only be constructed from a combination of polar and axial vectors, and would therefore be vanishing in the absence of stratification and rotation. The $\eta_{i j}$ tensor, on the other hand, does not require this, and it should be finite even in the completely homogeneous case. This is the case considered in the present study, which is a necessary intermediate step.

For homogeneous flows $\eta_{i j}$ is an isotropic tensor, which we write as $\eta_{i j}=\eta_{\mathrm{t}} \delta_{i j}$, where $\eta_{\mathrm{t}}$ is the turbulent magnetic diffusivity. A relevant concern in mean-field theory is that turbulent transport coefficients such as $\eta_{\mathrm{t}}$ must stay finite even in the limit of large values of the magnetic Reynolds number, defined here as $R_{\mathrm{m}}=u_{\mathrm{rms}} / \eta k_{\mathrm{f}}$, where $u_{\mathrm{rms}}$ is the rms velocity of the turbulence, and $k_{\mathrm{f}}$ is the wavenumber corresponding to the scale of the energy-carrying motions. Given the importance of a possible $R_{\mathrm{m}}$ dependence, it is necessary to perform so-called direct simulations, where no subgrid scale modeling is used. This implies that we must make compromises regarding the strength of the forcing and consider only subsonic flows. Following earlier work of Mee \& Brandenburg (2006) we consider a flow driven by random expansion waves of radius $R=2 / k_{\mathrm{f}}$ (not to be confused with the magnetic Reynolds number $R_{\mathrm{m}}$ ) and determine $\eta_{\mathrm{t}}$ using the test-field method of Schrinner et al. (2005) in the implementation of Brandenburg (2005).

The evolution of internal energy and hence entropy is not relevant to our question about turbulent transport coefficients. Therefore we consider an isothermal equation of state where the pressure $p$ is proportional to the density $\rho$ with $p=\rho c_{\mathrm{s}}^{2}$, with $c_{\mathrm{s}}$ being the isothermal speed of sound. We adopt a Gaussian potential forcing function $f_{\mathrm{f}}$ of the form $F_{\mathrm{f}}(\boldsymbol{x}, t)=\boldsymbol{\nabla} \phi$ with $\phi(\boldsymbol{x}, t)=N \exp \left\{\left[\boldsymbol{x}-\boldsymbol{x}_{\mathrm{f}}(t)\right]^{2} / R^{2}\right\}$, where $\boldsymbol{x}=(x, y, z)$ is the position vector, $\boldsymbol{x}_{\mathrm{f}}(t)$ is the random forcing position, $R$ is the radius of the Gaussian, and $N$ is a normalization factor. We consider a time dependence of $\boldsymbol{x}_{\mathrm{f}}$ with a forcing time $\delta t_{\mathrm{force}} \approx\left(u_{\mathrm{rms}} k_{\mathrm{f}}\right)^{-1}$ that defines the interval during which $\boldsymbol{x}_{\mathrm{f}}$ remains constant. We use the PENCIL Code (http://pencilcode.googlecode.com) which is a non-conservative, high-order, finite-difference code (sixth order in space and third order in time) for solving the compressible hydrodynamic and hydromagnetic equations. 


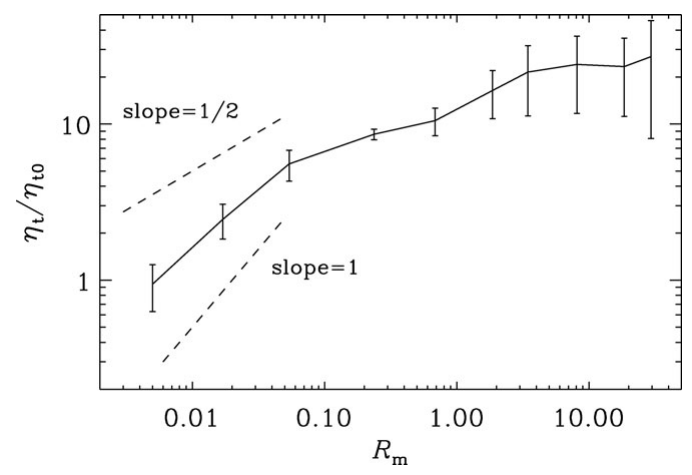

Figure 1. Dependence of $\eta_{\mathrm{t}}$ on $P_{m}=1$.

In Fig. 1 we plot the dependence of $\eta_{\mathrm{t}}$ on $R_{\mathrm{m}}$. Following earlier work of Sur et al. (2008) we normalize $\eta_{\mathrm{t}}$ by $\eta_{\mathrm{t} 0} \equiv u_{\mathrm{rms}} / 3 k_{\mathrm{f}}$. Note that, for low values of $R_{\mathrm{m}}, \eta_{\mathrm{t}}$ increases proportional to $R_{\mathrm{m}}^{n}$ with $n$ between $1 / 2$ and 1 . For larger value of $R_{\mathrm{m}}, \eta_{\mathrm{t}}$ seems to levels off at a value of about 20 times $\eta_{\mathrm{t} 0}$. Expressing this in terms of $u_{\mathrm{rms}}$ and the typical radius $R$ of the expansion waves, we find that $\eta_{\mathrm{t}} \approx 4 u_{\mathrm{rms}} R$. Note also that $\eta_{\mathrm{t}}$ is always positive, in contrast to analytic predictions for irrotational turbulence using the first-order smoothing approximation (Krause \& Rädler 1980).

Based on these results we can conclude that nearly irrotational turbulence is at least as efficient as vortical turbulence in diffusing mean magnetic field. Clearly, our study is still at a preliminary stage. It is important to clarify a possible dependence of our results on the microscopic magnetic Prandtl number and, in the nonlinear regime, on the magnetic field strength. Next, we need to consider the case with rotation and stratification which should then lead to an $\alpha$ effect, as well as turbulent pumping. This would provide an opportunity to compare with early predictions by Ferrière (1992) for this type of flows.

\section{Acknowledgements}

We acknowledge the allocation of computing resources provided by the Swedish National Allocations Committee at the Center for Parallel Computers at the Royal Institute of Technology in Stockholm and the National Supercomputer Centers in Linköping. This work was supported in part by the European Research Council under the AstroDyn Research Project 227952 and the Swedish Research Council grant 621-2007-4064.

\section{References}

Brandenburg, A. 2005, Astron. Nachr., 326, 787

Ferrière, K. 1992, ApJ, 389, 286

Krause, F. \& Rädler, K.-H. 1980, Mean-field magnetohydrodynamics and dynamo theory (Pergamon Press, Oxford)

Mee, A. J. \& Brandenburg, A. 2006, MNRAS, 370, 415

Schrinner, M., Rädler, K.-H., Schmitt, D., et al. 2005, Astron. Nachr., 326, 245

Sur, S., Brandenburg, A., \& Subramanian, K. 2008, MNRAS, 385, L15 